\title{
PROPUESTA DE EXPLOTACIÓN DE UN CORPUS ELECTRÓNICO AD HOC EN LA CLASE DE TRADUCCIÓN ESPECIALIZADA
}

Proposal of exploitation of an ad hoc electronic corpus in specialized translation courses

\section{Lucila María Pérez Fernández*}

\begin{abstract}
RESUMEN
Tras comprobar una bajada drástica en la calidad de las traducciones de los alumnos que se enfrentaban por primera vez a la asignatura de Introducción a la traducción especializada, se decidió llevar a cabo un experimento con el objetivo de evaluar la eficacia del uso de un corpus electrónico comparable ad hoc creado por los propios estudiantes, con el fin de mejorar tanto la calidad de sus traducciones como el proceso de aprendizaje de la traducción especializada en general. Este estudio se desarrolló en cinco fases: la fase 1, en la que los alumnos se familiarizan con el lenguaje especializado; la fase 2 de análisis del encargo de traducción y búsqueda de documentación de fuentes fiables y creación del corpus ad hoc; la fase 3 en la que se analiza el corpus y se extrae la terminología mediante glosarios; la fase 4 de explotación del corpus en un encargo específico y la fase 5 en la que los alumnos evalúan mediante un cuestionario la utilidad del corpus con respecto a su método de búsqueda terminológica de manera habitual.
\end{abstract}

Palabras clave: corpus electrónico, lingüística de corpus, traducción especializada, didáctica de la traducción, terminología.

\begin{abstract}
After verifying a dramatic drop in the quality of student's translations facing the subject "Introduction to specialized translation" for the first time, we decided to implement an experiment with the aim of evaluating the usefulness of an ad hoc electronic corpus created by the students themselves. The objective was to improve not only the quality of their translations but also their capacity to translate specialized texts. This study took place in five different stages: phase 1, familiarising with specialized language; phase 2, analysis of the scope of the translation project, search of reliable sources and creation of the ad hoc corpus; phase 3, corpus analysis and extraction of terminology; phase 4, exploitation of corpus and phase 5, evaluation of the whole process by answering a questionnaire where students evaluate the usefulness of corpora compared to their usual terminology research methods.
\end{abstract}

Key Words: Electronic corpus, corpus linguistics, specialized translation, translation didactics, terminology. 


\section{Introducción}

El salto de las asignaturas de traducción general a la traducción especializada constituye un gran desafío para los alumnos del grado de Traducción e Interpretación, pues de pronto comprueban cómo los métodos de búsqueda de terminología que venían usando hasta el momento no son suficientemente completos para afrontar con éxito los problemas de traducción que van surgiendo.

En este sentido, consideramos que un corpus electrónico ad hoc se revela como una valiosa fuente de información documental $\mathrm{y}$ terminológica en el marco de la enseñanza de traducción especializada.

La Traducción Especializada Inglés/ Español en el Grado en Traducción e Interpretación de nuestra universidad se divide en dos asignaturas cuatrimestrales de 6 créditos cada una y 4 horas de clase presenciales a la semana. Para la elaboración de este artículo nos centraremos en la primera de ellas cursada el $2^{\circ}$ año y que aborda la traducción de textos jurídicos y económicos.

El objetivo de este estudio es evaluar la eficacia del uso de un corpus electrónico comparable ad hoc creado por los propios estudiantes, con el fin de mejorar tanto la calidad de sus traducciones como el proceso de aprendizaje de la traducción especializada en general. Esta idea surgió tras comprobar una bajada drástica en la calidad de las traducciones de los alumnos que se enfrentaban por primera vez a la asignatura de Introducción a la traducción especializada, pues los métodos de búsqueda de correspondencias terminológicas que habían venido utilizando hasta el momento no cubrían todas las necesidades de los encargos de esta naturaleza.

Para llevar a cabo el objetivo de nuestro estudio se diseñó un experimento llevado a cabo con un grupo compuesto de 28 estudiantes y que se desarrolló en cinco fases: la fase 1, en la que los alumnos se familiarizan con el lenguaje especializado; la fase 2 de análisis del encargo de traducción y búsqueda de documentación de fuentes fiables y creación del corpus ad hoc; la fase 3 en la que se analiza el corpus y se extrae la terminología mediante glosarios; la fase 4 de explotación del corpus en un encargo específico y la fase 5 en la que los alumnos evalúan mediante un cuestionario la utilidad del corpus con respecto a su método de búsqueda terminológica de manera habitual.

Como indica Andrades (2013: 3), actualmente la compilación de un corpus constituye una herramienta terminológica y de documentación imprescindible en la mayoría de los trabajos de traducción, por lo que mediante este estudio pretendemos también acercar al alumnado de Traducción e Interpretación a la realidad del mundo profesional.

\section{Competencias del traductor especializado}

De acuerdo con Tolosa (2013:121), en el ámbito de la traductología empieza a hablarse de competencia a partir de los años 80 y desde entonces muchos han sido los autores (Bell, 1991; Hurtado, 1996; Hansen, 1997; el grupo PACTE, 2001, 2003 y 2005, entre otros) que han tratado de definirla, analizar los elementos que la componen e incluso proporcionar diferentes tipologías para su clasificación o diferentes análisis en función de los ámbitos de especialidad que se quieran estudiar.

Corpas (2004a: 138) analiza la variación de las subcompetencias traductoras en el ámbito biosanitario y concluye que si bien las subcompetencias translativa y profesional no parecen experimentar una variación considerable, las subcompetencias lingüísticoculturales, textuales y documentales del traductor especializado sí presentan matices diferentes. Para nosotros, este estudio es perfectamente extrapolable al ámbito jurídico que aquí nos ocupa. En primer lugar, consideramos que la subcompetencia lingüística-cultural se ve alterada con respecto a la de un traductor general, pues requiere conocer las peculiaridades y diferencias existentes en el lenguaje jurídico de las dos lenguas de trabajo, así como conocimientos suficientes de la terminología 
y unidades fraseológicas propias del ámbito de especialidad concreto. Además, requiere también el conocimiento de los dos sistemas legales en los que se enmarcan las lenguas de trabajo, pues muchas veces un concepto en LO puede presentar matices muy diferentes en su equivalente en LM o incluso directamente no existir. En segundo lugar, la subcompetencia textual que se encuentra muy relacionada con la anterior, pues el traductor necesitará ser consciente de las convenciones textuales que presenta cada tipo de texto (certificados académicos, sentencias de divorcio, etc.) y de las propias de cada lengua. Por último, llegamos a la subcompetencia documental que, como indica Hurtado (2001: 62), constituye el eje central del proceso traductor, pues comprende el uso de fuentes que le permitirán adquirir los conocimientos sobre el campo temático en cuestión y mejorarán la calidad del producto final.

Todas estos matices diferentes que caracterizan las competencias y subcompetencias del traductor especializado hacen que el corpus electrónico se presente como un instrumento muy eficaz a la hora de identificar la terminología y las estructuras fraseológicas de un determinado lenguaje de especialidad $y$, por lo tanto, constituya una herramienta de gran utilidad en las clases de traducción especializada.

\section{Explotación didáctica del corpus}

Antes de llevar a cabo el experimento, decidimos introducir una actividad de traducción inicial para comprobar si efectivamente los alumnos identificaban dificultades a la hora de enfrentarse a un encargo especializado y en caso afirmativo saber cuáles serían las más señaladas por la mayoría de ellos.

Como ya indicamos, el grupo de alumnos con el que trabajamos no se había enfrentado con anterioridad a ningún encargo de traducción especializada, por lo que consideramos interesante ponerlos en la situación para que fuesen conscientes de las carencias que presentan las obras lexicográficas generales a la hora de buscar equivalentes en contextos no generales.
El texto escogido para este primer acercamiento fue una sentencia del Tribunal de Justicia europeo. Los alumnos realizaron este encargo por parejas y añadieron un comentario sobre las dificultades principales que habían identificado. Entre todas las señaladas identificamos tres problemas recurrentes:

- la búsqueda de equivalentes a la terminología especializada,

- la abundancia de términos polisémicos y, en consecuencia, la dificultad de escoger la acepción más adecuada,

- la abundancia de unidades y fórmulas fraseológicas propias del lenguaje jurídico, para las que, a veces, ni los diccionarios especializados ofrecen propuesta alguna.

Una vez identificadas las dificultades principales que habían experimentado, pusimos en marcha nuestro proyecto de explotación didáctica del corpus en el aula de traducción jurídica, para lo cual se diseñó un experimento que se llevó a cabo en cinco fases.

\subsection{Fase 1: familiarización con el lenguaje especializado}

Una de las mayores dificultades a las que deben hacer frente los estudiantes de traducción jurídica (cuyos conocimientos del ámbito jurídico suelen ser prácticamente nulos) es a producir textos que se ajusten a las convenciones propias del discurso jurídico; por eso, consideramos relevante en esta primera fase dedicar dos unidades al estudio del lenguaje jurídico inglés y del lenguaje jurídico español, respectivamente.

Esta fase se llevó a cabo a lo largo de dos semanas, en un total de ocho horas distribuidas en cuatro sesiones (dos sesiones de dos horas de duración por semana). La primera se dedicó al estudio de los rasgos propios del inglés jurídico (unidad 1) y la segunda al español jurídico (unidad 2).

Para ello se diseñó un cuadernillo llamado "Manual de apoyo", en el que se incluyó una gran multitud de actividades para llevar a cabo dentro y fuera del aula y que abarcaban el 
estudio del léxico jurídico, la abundancia de latinismos, el estilo arcaizante, las colocaciones, los eufemismos, los tecnicismos, los cultismos, las locuciones, la ambigüedad sintáctica, las abreviaturas y las siglas.

Al concluir esta fase se les pidió a los alumnos la elaboración de un ejercicio práctico consistente en la comprensión de una sentencia del Tribunal de Justicia europeo redactada en español y con una estructura muy similar a la que se les había encargado traducir en la fase inicial. El objetivo de esta actividad era no solo comprobar la efectividad de las sesiones dedicadas al estudio del lenguaje especializado, sino que los alumnos descubriesen por sí mismos la utilidad de disponer de un banco de textos paralelos a la hora de enfrentarse a determinados encargos de traducción especializada.

\subsection{Fase 2: Análisis del encargo, documentación y creación del corpus}

Una vez que los alumnos se habían familiarizado con el lenguaje jurídico, el docente entregó el encargo de traducción para el cual se iba a crear el corpus. En este caso se trataba de la traducción de un contrato de licencia de patente (patent license agreement) escrito originalmente en inglés.

De entre los distintos tipos posibles de corpus se optó por uno ad hoc, definido por Corpas (2004b: 236) como "un corpus virtual que se compila puntualmente para la realización de un determinado encargo de traducción en cualquier dirección (directa, inversa o indirecta)." y de carácter comparable que, de acuerdo con Corpas (2004b: 153), consiste en una compilación de textos originales en la lengua meta equiparables al $\mathrm{TO}$ en cuanto a su temática, nivel de especialización, situación comunicativa y tipología textual.

Como indica Castillo (2009: 3) antes de comenzar la fase de documentación es conveniente establecer los criterios específicos que regirán la creación del corpus. Para ello, nos basamos en los establecidos por Bowker y Pearson (2002: 54): propósito del corpus, tamaño, medio, temática, tipo textual, autoría, fecha de publicación y lengua/s.

a) el propósito de la compilación: recopilar textos en la LM que facilitasen la comprensión del TO y ayudasen a producir un TM de calidad.

b) el tamaño: dado que se trataba de un corpus dirigido a la realización de un encargo específico para el cual contaban con una semana de plazo establecimos un mínimo de 10 textos.

c) el medio: escrito, en formato electrónico.

d) la temática: aunque en la clase lo habitual es que la temática varíe cada semana, en este encargo concreto nos centramos en la recopilación de contratos.

e) el tipo textual: buscamos en este caso tipos de textos cuya función fuese la misma que el TO, por lo que la búsqueda específica se centró en la recopilación de licencias de patentes.

f) la autoría: con el fin de que el grado de fiabilidad fuese elevado los textos incluidos en el corpus debían proceder de centros de investigación, universidades o portales de Internet en los que se ofrecen muestras de documentos jurídicos.

g) la fecha de publicación: se puso la condición de intentar, en la medida de lo posible, que los textos no tuviesen una antigüedad mayor a siete años.

h) la/s lengua/s: nos ceñimos a los documentos redactados en español peninsular.

Una vez establecidos todos los criterios generales y específicos de creación del corpus, los alumnos comenzaron la fase de documentación en la que se centraron en la búsqueda de documentos digitales exclusivamente en línea, pues la compilación daría lugar a un corpus electrónico. 


\subsection{Fase 3: Análisis del corpus y extracción de terminología}

Para el análisis del corpus utilizamos la herramienta de software libre AntConc, por tratarse de una herramienta sencilla de usar, que permite identificar patrones de uso gramatical $o$ frases que se repiten con frecuencia y a la que los alumnos pueden tener acceso en sus propios equipos, pues cuenta con versiones para diferentes sistemas operativos.

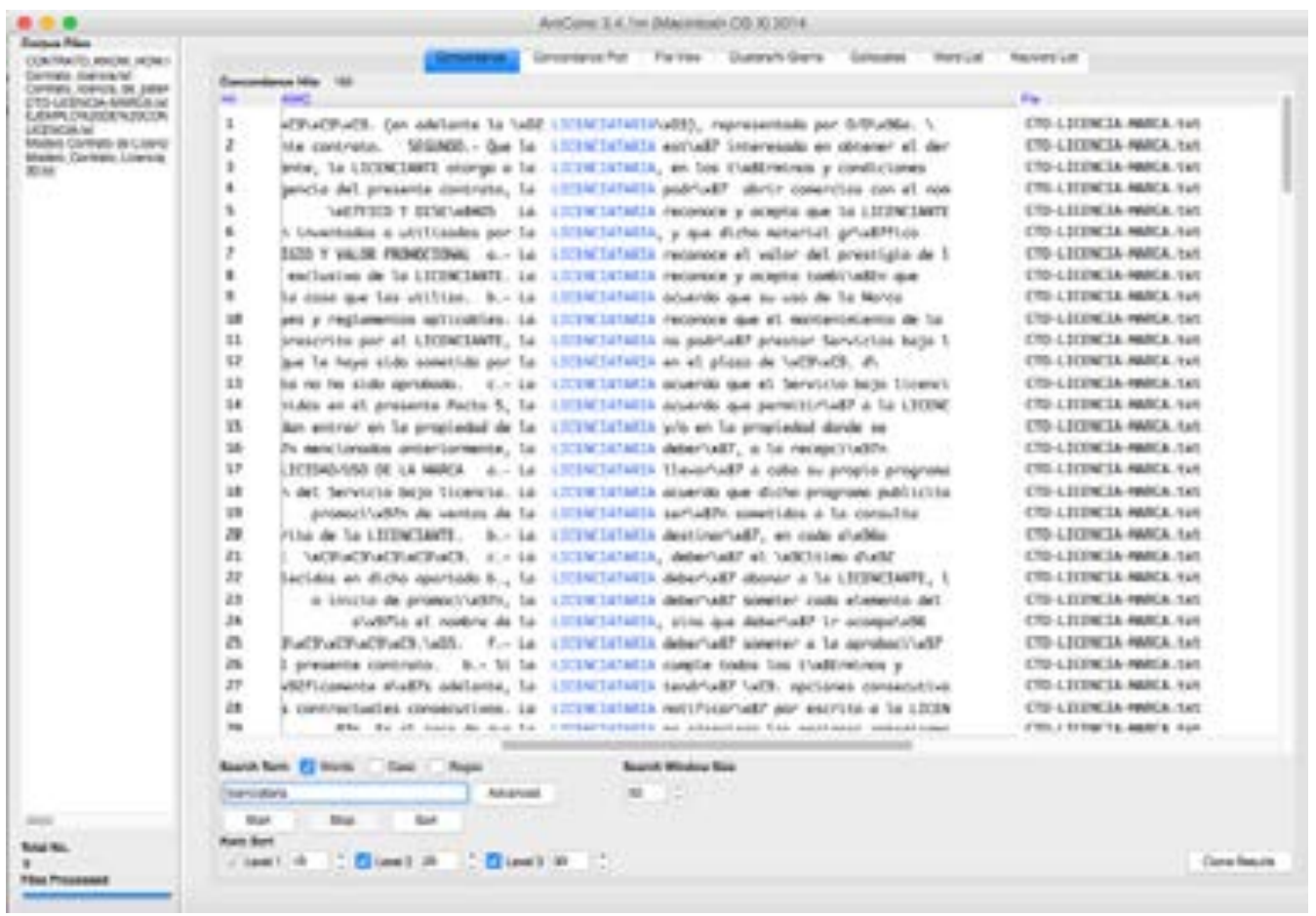

Una vez compilado el corpus, los alumnos extraen la terminología y crean glosarios que luego podrán además usar en Programas de Traducción Asistida o en las asignaturas de Interpretación. Aunque el programa AntConc cuenta con una herramienta llamada WordList, que permite crear un listado de palabras partir de todos los textos seleccionados, cabe señalar que en nuestra universidad contamos con una plataforma virtual, que incluye una herramienta de creación de glosarios, que permite añadir definiciones o indicaciones contextuales para cada uno de los términos, por lo que esta es la herramienta que usamos con mayor frecuencia.

\subsection{Fase 4: Explotación del corpus en el encargo específico de traducción}

En esta fase los alumnos llevaron a cabo la traducción del documento original con ayuda del corpus compilado. Se dedicaron dos sesiones de dos horas cada una para traducir y una tercera sesión dedicada a la revisión.

Una vez finalizado el encargo, se dedicó una sesión para poner en común los trabajos y efectuar una corrección grupal, permitiendo que los alumnos ofreciesen posibles soluciones a los diferentes problemas de traducción. 
Los alumnos culminaron esta fase alineando el texto original y la traducción realizada por ellos mismos con el fin de crear una memoria de traducción de Trados, que se irá alimentando con posteriores traducciones realizadas en la asignatura, pues se persigue que todo el trabajo que lleven a cabo en clase sirva para que cada uno cree y disponga de un recurso que les será útil cuando finalicen sus estudios y quieran incorporase al mundo laboral.

\subsection{Fase 5. Cuestionario de autoevaluación}

Finalmente, se entrega a los alumnos un cuestionario anónimo de autorreflexión, en el cual deberán valorar la utilidad de la tarea realizada con respecto al método de búsqueda terminológica que venían realizando hasta ahora de manera habitual (principalmente la consulta de obras lexicográficas monolingües y bilingües).

En este cuestionario debían señalar de cero a cinco, siendo cero la puntuación más baja y cinco la más alta, su acuerdo o desacuerdo con las siguientes afirmaciones:

1. La creación de los corpus ha contribuido a reducir el tiempo empleado para realizar la traducción.

2. La creación del corpus ha contribuido a mejorar la calidad de mis traducciones.

3. La creación del corpus ha contribuido a mejorar mi proceso de aprendizaje de la traducción especializada.

4. Mediante el corpus creado he mejorado mi aprendizaje de la lengua extranjera.

5. Considero que la compilación del corpus y creación de los glosarios me serán útiles en otras asignaturas o en futuros encargos de traducción.

\section{Conclusión y análisis de resultados}

Mediante este estudio hemos querido evaluar la eficacia del uso de un corpus comparable ad hoc en la clase de traducción especializada inglés-español, concretamente en la parte de la asignatura que abarca la traducción de textos jurídicos.

Para ello hemos llevado a cabo un experimento dividido en cinco fases que han ido de la enseñanza de los lenguajes de especialidad en lengua origen y lengua término hasta la realización final de un encargo de simulación real, pasando por las fases de creación y análisis del corpus electrónico compilado.

Al término de la unidad didáctica los alumnos respondieron a un cuestionario anónimo de autorreflexión en el que valoraron positivamente el uso del corpus para la mejora de la calidad de producción de textos en lengua meta, para la reducción del tiempo empleado en realizar la traducción, para reforzar el aprendizaje en las clases de traducción especializada, así como de la lengua extranjera en general. Asimismo, todos los alumnos afirmaron ver la utilidad de la creación de glosarios a partir del corpus, en cuanto que podrían usarlos en otros encargos de la misma asignatura, así como en las clases de Interpretación cuando se traten discursos de temática similar.

Por lo tanto, tras analizar los resultados obtenidos a partir del cuestionario, podemos concluir que se comprueba la eficacia del uso de un corpus comparable ad hoc como herramienta didáctica en la clase de traducción especializada, puesto que contribuye positivamente a mejorar la competencia traductora de los estudiantes.

\section{Referencias bibliográficas}

Andrades Moreno, A. 2013. "Internet como fuente para la compilación de corpus jurídicos". En: Enlaces: revista del CES Felipe II (15): 1-15.

Bell, R. 1991. Translation and Translating. Londres y Nueva York: Longman.

Bowker, L. y J. Pearson. 2002. Working with Specialized Language: A practical guide to using corpora. Londres: Routledge. 
Castillo Rodríguez, C. 2009. "La elaboración de un corpus ad hoc paralelo multilingüe". En: Revista Tradumática: Traducció $i$ Tecnologies de la Informació $i$ la Comunicació, 7. Recuperado de http:// ddd.uab.cat/pub/tradumatica/15787559n7/ 15787559n7a3.pdf. Consulta: 27-07-2017

Corpas Pastor, G. 2004a. "La traducción de textos médicos especializados a través de recursos electrónicos y corpus virtuales." En: Las palabras del traductor. Actas del II Congreso Internacional "El español, lengua de traducción”, 20 y 21 de mayo, Toledo 2004. L. González y P. Hernúñez (eds.): 137-164. Bruselas: Comisión Europea/ESLETRA. Recuperado de http://cvc.cervantes.es/lengua/esletra/ pdf/02/017 corpas.pdf. Consulta: 27-07$\underline{2017}$

Corpas Pastor, G. 2004b. "Localización de recursos y compilación de corpus via internet: aplicaciones para la didáctica de la traducción médica especializada". C. Gonzalo García - V. García Yebra (eds.). En: Manual de documentación y Terminología para la traducción especializada. Madrid: Arco: 223-258.
Hansen, G. 1997. "Success in translation." En: Perspectives: Studies in Translatology 5 (2): 201-10.

Hurtado Albir, A. 1996. La enseñanza de la traducción. Castellón de la Plana: Universitat Jaume I.

Hurtado Albir, A. 2001. Traducción y traductología: introducción a la traductología. Madrid: Cátedra.

PACTE. 2001. La competencia traductora y su adquisición. En: Quaderns 6: 39-45.

PACTE. 2003. Building a translation competence model. F. Alves (Ed.). En: Triangulating Translation: 43-66. Amsterdam: John Benjamins.

PACTE. 2005. Investigating Translation Competence: Conceptual and Methodological Issues. En: Meta 50 (2): 609-619.

Tolosa Igualada, M. 2013. Don de errar. Tras los pasos del traductor errante. Castelló de la Plana: Publicacions de la Universitat Jaime I.

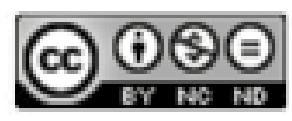

Este obra está bajo una licencia de Creative Commons Reconocimiento-NoComercial-SinObraDerivada 4.0 Internacional. 